\title{
Effect of Power Control on D2D Cellular and LED Communications
}

\author{
Soo-Hyeong Kang and Jeong Gon Kim* \\ Department of Electronic Engineering, Korea Polytechnic University \\ soo7586@naver.com,jgkim@kpu.ac.kr
}

\begin{abstract}
Recent development of various devices and communication technologies has not only increased long distance communication-short distance communication technology is gradually increasing as well. Among the short-range communication technologies, D2D (Device-to-Device) communication facilitates direct communication between devices without a base station. D2D technology will be able to work with short-distance communication technologies such as Bluetooth, Wi-Fi, Zigbee, and LED communications to achieve higher efficiency. In particular, LED communication, which utilizes LEDs, is being developed for use in various applications such as vehicles and indoor and outdoor applications, to name a few. D2D technology that utilizes LED communication will show higher efficiency because it uses a wide and fast frequency band for LED communication, in addition to cellular frequency resources. Although D2D communication reduces the load on the base station through direct communication, it also causes interference through the sharing of cellular frequency. Problems caused by interference are a disadvantage, and can occur when using LED communication as well as cellular frequencies. In this paper, we have researched algorithms that allocate resources by using location information to eliminate interference caused by D2D sharing resources. We propose to add power control to the resource allocation algorithm that utilizes location information to improve the efficiency of interference cancellation. This power control is also a key factor in reducing the amount of power required to take advantage of future LED communications.
\end{abstract}

Keywords: D2D, Cellular network, Uplink, Resource allocation, Power control, Li-Fi

\section{Introduction}

D2D communication is one of the short-range communication technologies available today. As the number of devices is increasing, communication between devices has become increasingly important in indoor and outdoor locations. As such, the need for D2D communication technology has increased in order to efficiently allocate frequency resources to the growing number of devices. Device-to-device communication (D2D) has been announced as a key technology in LTE-Advance networks [1]. The advantages of D2D communication over traditional cellular transmission include not only the proximity gain in terms of improved link budget, but also the so-called re-use and hop gains [2]. It is also available in combination with cellular networks as well as other communication technologies. When cellular networks are not available, D2D communication can be used in combination with technologies such as VLC (Visible Light Communication). As the demand for wireless communications is growing despite the saturation of the RF spectrum, VLC becomes a viable option as it provides worldwide, unregulated and almost unlimited bandwidth. Even in its early-stages, VLC allows for short range, multi $\mathrm{Gb} / \mathrm{s}$

Received (January 5, 2018), Review Result (March 9, 2018), Accepted (March 12, 2018)

* Corresponding Author 
data rates [3]. In other words, it is possibility to maintain and develop D2D communication using other communication technologies, such as LED communications as well as cellular networks. D2D communication can be used in vehicles as well as indoor and outdoor applications as it can be converged with other communication technologies. D2D can be used to allocate resources for vehicle communications and can provide proximity-based commercial services, such as advertising and marketing, SNS(Social Networking Service) [4][5].

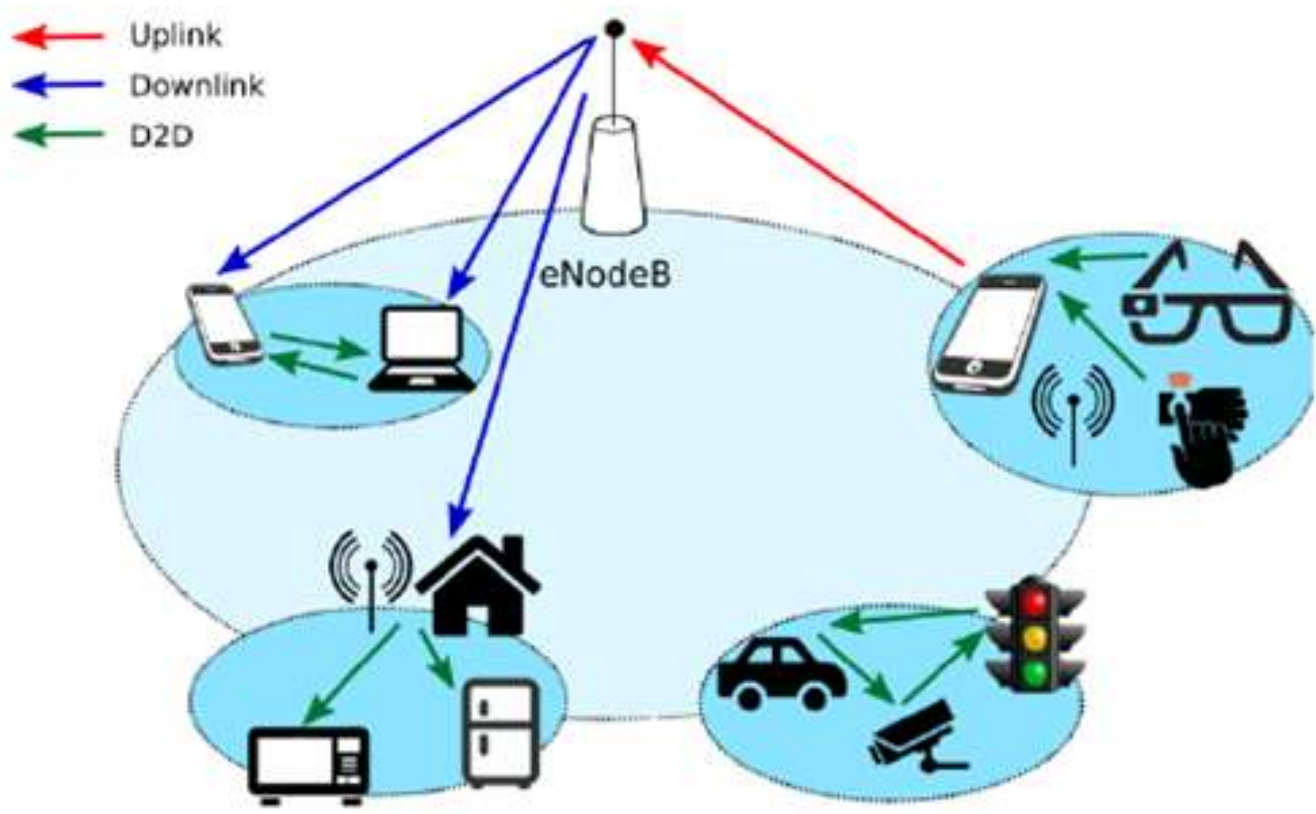

Figure 1. D2D Communication Concept

D2D communication is a technology in which two devices communicate directly without using a base station, which reduces the load on the latter. In addition, as it can be used by sharing other frequency resources, such as the frequencies of cellular communications, LED communications, and license-exempted bands, it is not necessary to have a dedicated frequency for D2D communication However, as different communication frequency bands are shared, interference between devices using the same resources occurs, and is likely a fatal factor as it not only degrades the QoS (Quality of Service) of the D2D communication, but it also reduces the QoS of the communication technology sharing the resources. There are three cases of interference in the uplink environment: First, interference occurs in the existing communication technology devices on the D2D link. In this case, when using D2D communication, the signal received by the base station of the existing communication technology affects the D2D link. Second, interference occurs between D2D links and the base stations using existing communication technologies. In this case, the D2D link is added when the existing communication technology is already in use, and the base station that uses the existing communication technology is affected by the interference. Third, interference occurs between D2D links. This happens when two or more D2D links are using the same resource. As a result, the QoS between the D2D links is diminished.

Methods for reducing interference caused by D2D communication have been and will continue to be studied. A typical method for reducing interference includes a scheme that combines resource allocation and power control. Several techniques have been proposed to optimize performance by using SINR (Signal-to-Interference-plus-Noise Ratio) technology and power control technology, as a way to reduce interference to the 
surrounding environment. Examples include a technique that cannot allocate resources if the minimum SINR is not satisfied by calculating the SINR, and a technique in which the SINR value is considered in the multicast environment and the power value is derived according to the considered environment [6][7]. Further, a technique for power control through analyzing SINR by allocating resources based on area information has been reported [8]. Other technologies have confirmed that higher efficiency can be achieved if power control is performed at the same time when allocating resources. It is confirmed that the efficiency increases when the power control is performed simultaneously with the resource allocation algorithm using both the location and the SINR information.

In this paper, we assume that the D2D link shares resources with the cellular network. The D2D link uses the location information and the SINRs in the cellular network to find the maximum data rate and to allocate resources. The number of resources in a cellular network has the same number of resources as a device in a cellular network. It is also assumed that the base station knows the location information of all devices through GPS. Resource allocation is done in two steps: First, the location information is used to create a resource group, to be used by the D2D link as the available resources among the cellular resources. Second, the D2D link selects and allocates resources using the SINR so that the maximum data rate is selected among the selected resource groups. Power control is performed with a simple algorithm, which results in either a power up or a power down, in order to satisfy the minimum SINR. We added location-sensitive power control to reduce power loss in VLC, by increasing the power efficiency in cellular and D2D communications before embodying LED Communication.

\section{Related System}

\subsection{LED Communication}

Visible LED communication (VLC), which utilizes LED illumination for signal transmission, offers a wide range of unlicensed, high capacity spectrum resources. It has been considered a promising technology when collaborated with the radio frequency communication networks in the heterogenous networks [9]. VLC is ubiquitous and radiation-free, to name some of its attractive features. However, due to the natural property of the visible LED propagation and its intensity modulation scheme, severe coverage problems occur when the visible LED signal is blocked or interfered by strong sun-LED. To mitigate this issue, the combination of cellular communication networks with the VLC network has been proposed [10]. As a result of this method, the cellular communication network is able to control and guarantee the coverage of data transmission, while the VLC network can provide high speed data transmission for wireless users. However, even though the cellular communication network guarantees the coverage, the spectrum and energy efficiency are poor during the services. Zhang et al. [11] assumed that the VLC network could be accessed by combining the VLC network with the D2D technology. In addition, a few mobile users have proposed a way to relay the transmitted data to the surrounding mobile users, through representation of either shaded or strong sun-LED.

In a cellular network, VLC is fused with D2D communication, a method which not only increases the efficiency of the cellular network, but also enables communication using more frequencies, thus reducing both the size of the interference and the burden on the cellular network. In our opinion, VLC will be able to take advantage of D2D communication with LED, as well as of D2D relay. 


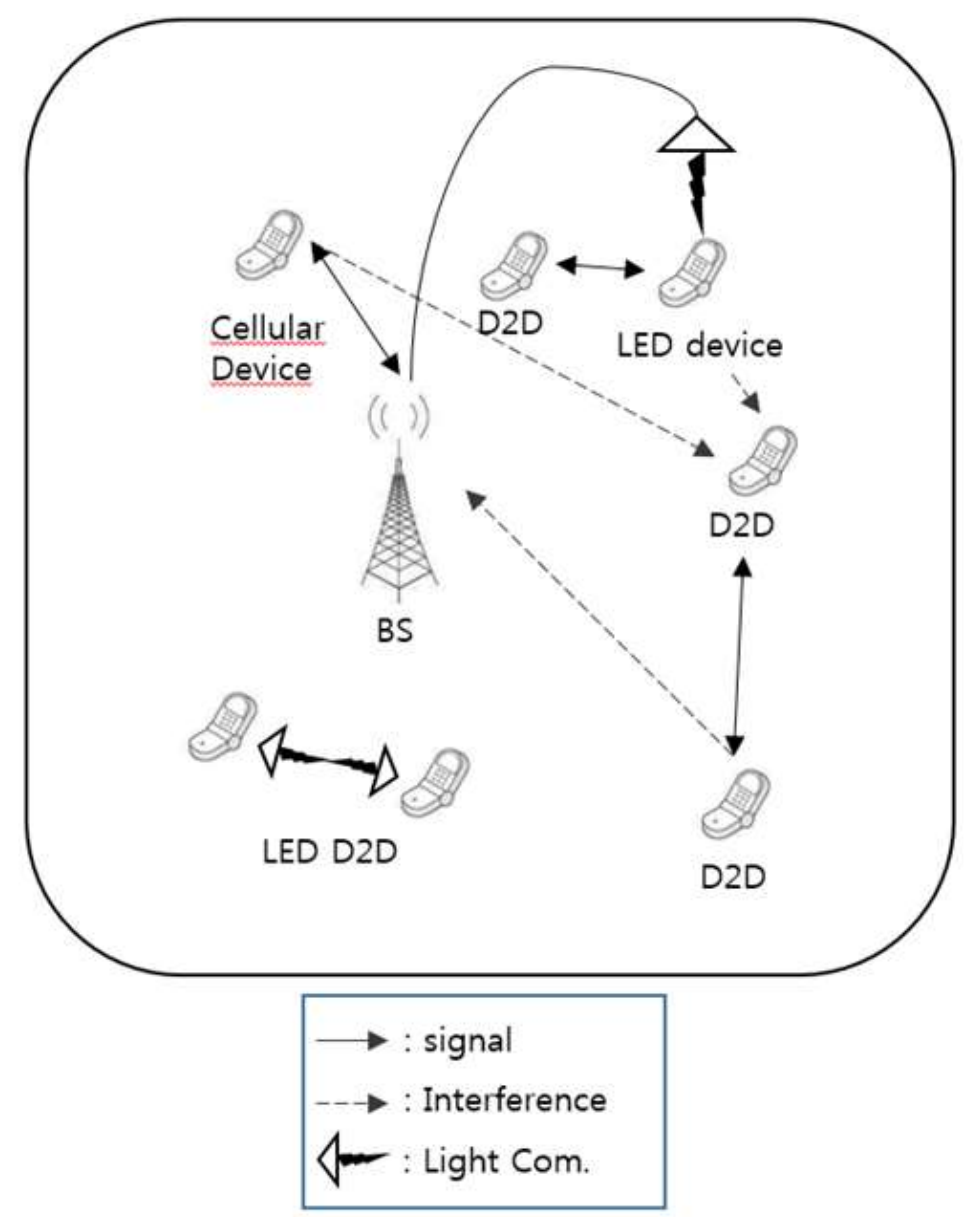

Figure 2. Combining D2D and LED Communication in a Cellular Network

\subsection{System Model}

Although several communication technologies exist, this paper assumes that the D2D link allocates resources in the cellular network. Cellular devices and D2D links share cellular resources in a single-cell scenario environment. The number of cellular resources is equal to the number of cellular devices, and each cellular device uses only one cellular resource. The same scenario applies for D2D links. The purpose of this paper is to show how the sum of the data rates of D2D links and cellular devices can be maximized in an environment where the same resources are shared.

The number of cellular resources existing in the cellular network is $\mathrm{N}$. The number of cellular devices is $\mathrm{C}$, and the number of $\mathrm{D} 2 \mathrm{D}$ links is $\mathrm{D}$. The cellular resource $\mathrm{N}$ is a resource group of $\{1, \ldots, n\}$. Cellular Device $C$ is a resource group of $\{1, \ldots, c\}$ and D2D link $\mathrm{D}$ is a resource group of $\{1, \ldots, d\}$.

The D2D link and the cellular device need to satisfy a certain SINR value. The SINR value to be satisfied by the cellular device is indicated as ' $r_{\text {cellular' }}$ and the SINR value to be satisfied by the D2D link is indicated as ' $\mathrm{r}_{\mathrm{D} 2 \mathrm{D}}$ '. The SINR equation to be satisfied is as follows:

$\mathrm{r}_{\text {cellular }}<$ SINR $_{\text {Cellular }}$

$\mathrm{r}_{\mathrm{D} 2 \mathrm{D}}<\operatorname{SINR}_{\mathrm{D} 2 \mathrm{D}}$ 
If the SINR value is not satisfied, it is assumed that the transmission has failed. Conversely, if the SINR value is satisfied, it is assumed that the transmission is successful. Finally, we define an equation to calculate the throughput to maximize the sum of the data rates of the D2D links and the cellular devices:

$$
\begin{aligned}
& \mathrm{R}_{\text {Cellular }}=\log _{2}\left(1+\operatorname{SINR}_{\text {Cellular }}\right), \\
& \mathrm{R}_{\mathrm{D} 2 \mathrm{D}}=\log _{2}\left(1+\operatorname{SINR}_{\mathrm{D} 2 \mathrm{D}}\right)
\end{aligned}
$$

where ' $R_{\text {Cellular' }}$ ' is the throughput of the cellular device and ' $R_{D 2 D}$ ' is the throughput of the D2D link. The sum of the maximum data rates is calculated as follows:

$$
\mathrm{R}_{\text {total }}=\sum_{\mathrm{m}=1}^{\mathrm{c}} \mathrm{R}_{\mathrm{m}}+\sum_{\mathrm{k}=1}^{\mathrm{d}} \mathrm{R}_{\mathrm{k}}
$$

The total data rate, $R_{\text {total }}$ is calculated by adding the sum of the set of cellular devices and the sum of the set of D2D links.

The SINR equation and a more detailed model of the system are described by Kang et al., [12].

\section{Proposed Scheme}

\subsection{Resource Allocation Scheme}

A method for allocating resources using only location information is used to find available resources by using location information through GPS, and to share one resource among available resources with D2D links [13]. In order to overcome the disadvantages caused by interference when using only location information, a resource allocation algorithm has been proposed[12], in which both the interference avoidance rate and the efficiency are increased through using both SINR and location information. In this paper, power control is performed based on a resource allocation algorithm that utilizes both location and SINR information.

As the resource allocation prior to the implementation of LED communication uses only cellular resources, the resource allocation algorithm comprises of only two simple steps: division into a cellular device selection algorithm for resource allocation, and a D2D link resource allocation algorithm.

\subsubsection{A Cellular Device Selection Algorithm for Resource Allocation}

Assuming that the base station knows the location of all devices, the distance between the cellular device and the D2D link can be calculated through the location information of the device. With the coordinates of the cellular device denoted by ' $\left(\mathrm{x}_{\mathrm{CUE}}, \mathrm{y}_{\mathrm{CUE}}\right)$ ' and the coordinates of the D2D link denoted by ' $\left(\mathrm{x}_{\mathrm{D} 2 \mathrm{DRX}}, \mathrm{y}_{\mathrm{D} 2 \mathrm{D} \mathrm{RX}}\right)^{\prime}$, the distance between the cellular device and the D2D link is calculated as follows:

$$
\mathrm{R}_{\mathrm{D} 2 \mathrm{D} \mathrm{RX}, \mathrm{CUE}}=\sqrt{\left(\mathrm{x}_{\mathrm{D} 2 \mathrm{D} \mathrm{RX}}-\mathrm{x}_{\mathrm{CUE}}\right)^{2}+\left(\mathrm{y}_{\mathrm{D} 2 \mathrm{D} \mathrm{RX}}-\mathrm{y}_{\mathrm{CUE}}\right)^{2}} .
$$

The base station calculates the distance to all cellular devices for one D2D link. The reason for this is that calculating the distance to the cellular device is the same as comparing the interference magnitudes of the resources, as the cellular device has assumed that each of the cellular devices has different resources in order to find the resources to be used by the D2D link. In the next step, we show how the base station aligns the distance between the D2D link and the cellular devices. As the interference 
between the D2D link and the cellular device is small as the distance increases, a resource that can be shared at only a certain rate among the distant resources is selected.

\subsubsection{A D2D Link Resource Allocation Algorithm}

After performing the cellular device selection algorithm for resource allocation process, each D2D link must find a cellular resource to share. In the case of resource allocation using only location information, interference between D2D links that occur when selecting the same resource is not considered. Therefore, it is necessary to calculate the SINR value in order to improve the interference. After performing the Resource Allocation scheme process, each D2D link must find a cellular resource to share. In the case of resource allocation using only location information, interference between D2D links that occur when selecting the same resource is not considered, so it is necessary to calculate the SINR value in order to improve the interference. To consider a D2D link using the same resource, the equation in SINR D2D should be expressed by the following equation.

$$
\operatorname{SINR}_{D 2 D}=\frac{P_{D 2 D} G_{D 2 D \text { Pair }}}{P_{\text {noise }}+\sum_{m=1}^{c} y_{m, k}\left(P_{\text {Cellular }} G_{m, k}+\sum_{i=1}^{d} y_{m, i} P_{D 2 D} G_{k, i}\right)},
$$

where ${ }^{\prime} G_{k, i^{\prime}}$ is the channel gain between other D2D links using the same resource and the current D2D link. After calculating the SINR of all cellular devices and the D2D link, we need to find the resource combination that yields the maximum data rate from the sum of the cellular device and the D2D links. When a resource combination is found, it is allocated to the D2D link.

As the amount of interference received by D2D or cellular is reduced when using VLC technology, a factor must be added to determine what resources the SINR equation uses. In other words, when VLC is used, it is at first necessary to select a cellular resource or a LED resource. After that, the resource group is selected, followed by resource allocation.

\subsection{Power Control}

The power control scheme consists of simple algorithms. To satisfy the minimum SINR value, the power of the D2D link is either increased or decreased to control the power.

If only the SINR of the D2D link is satisfied, the power of the D2D link is increased. Conversely, if the SINR of the cellular device is not satisfied, the power of the D2D link is reduced. If both are satisfied, the power allocation is finished. If both are unsatisfactory, the transmission is assumed to fail.

Power control is required in VLC because it is intended for use in real life. Too much LED is difficult to use in real life, so power control is required. In order to reduce the interference between LEDs, it is important to emit appropriate LED, so there is a high need for power control.

\section{Performance Evaluation}

The simulation environment as shown in Table 1 is designed using $\mathrm{C}++$. The number of cellular devices is 10 and the number of D2D links is seven. The minimum SINR that a cellular device must satisfy is $0 \mathrm{~dB}$. The minimum SINR that the D2D link must satisfy is $0 \mathrm{~dB}$ to $20 \mathrm{~dB}$. 
Table 1. Simulation Parameters

\begin{tabular}{|c|c|}
\hline Parameters & Value \\
\hline Cell radius & $500 \mathrm{~m}$ \\
\hline Maximum distance between D2D links in a pair & $50 \mathrm{~m}$ \\
\hline Minimum SINR of D2D link (Max.) & $40 \mathrm{~dB}$ \\
\hline Minimum SINR of D2D link (Min.) & $0 \mathrm{~dB}$ \\
\hline Minimum SINR of Cellular Device & $0 \mathrm{~dB}$ \\
\hline Maximum Transmission Power of D2D users & $13 \mathrm{dBm}$ \\
\hline Transmission Power of Cellular users & $23 \mathrm{dBm}$ \\
\hline Pnoise & $-114 \mathrm{dBm}$ \\
\hline Number of Cellular Device & 10 \\
\hline Number of D2D link & 7 \\
\hline Path loss & $128,1+37,6 * \log (\mathrm{d} / 1000)$ \\
\hline
\end{tabular}

Figure 5 shows the total, D2D, and cellular device throughputs when power control is applied. The total throughput for all cases shows an increase, except for the $10 \%$ case. a graph of throughput of the D2D link shows that rises as well as the overall throughput. The focus is on the D2D link because it is the power control that gradually increases the minimum D2D SINR. Conversely, the cellular device can see a decreasing trend. However, as the cellular device also satisfies the minimum SINR, communication is not impossible.

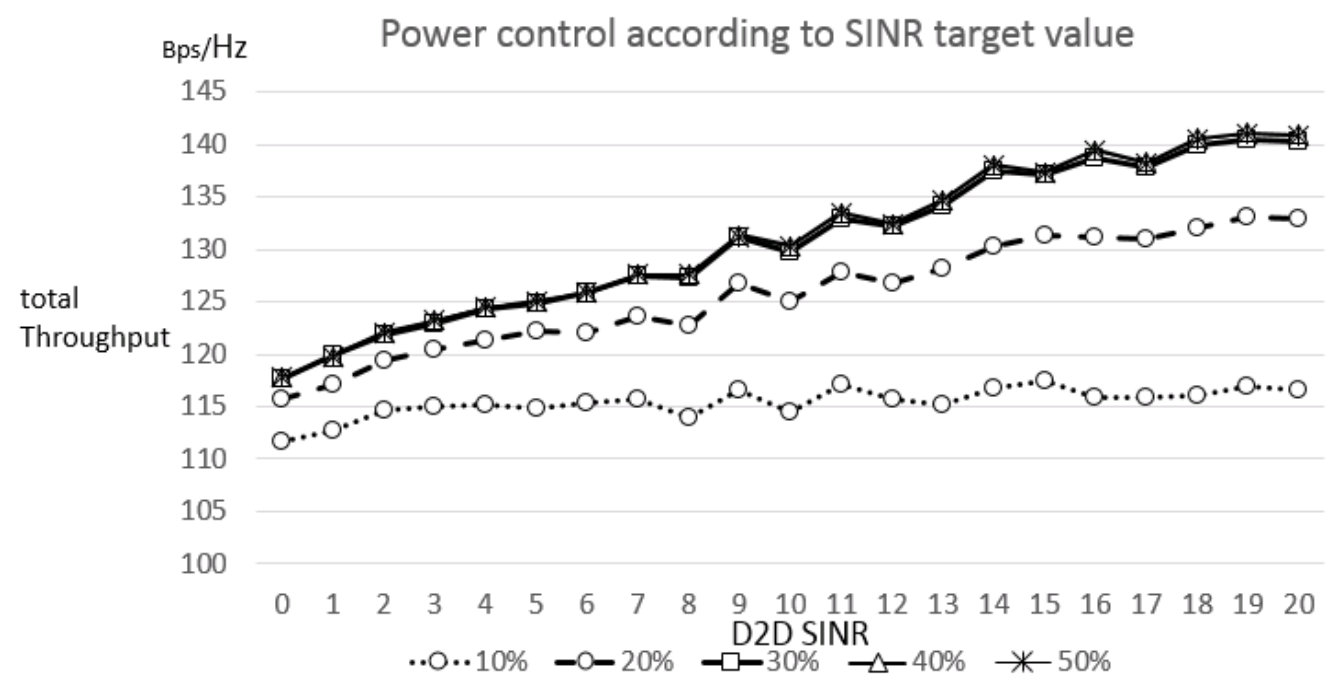

Figure 5. Throughput with Power Control According to SINR Target Value

Figure 6 shows the amount of power increase when the minimum SINR value of the D2D link is increased. As the only way to satisfy the minimum SINR value of the D2D link is to increase the power, the power is increased until the desired SINR value is achieved. 


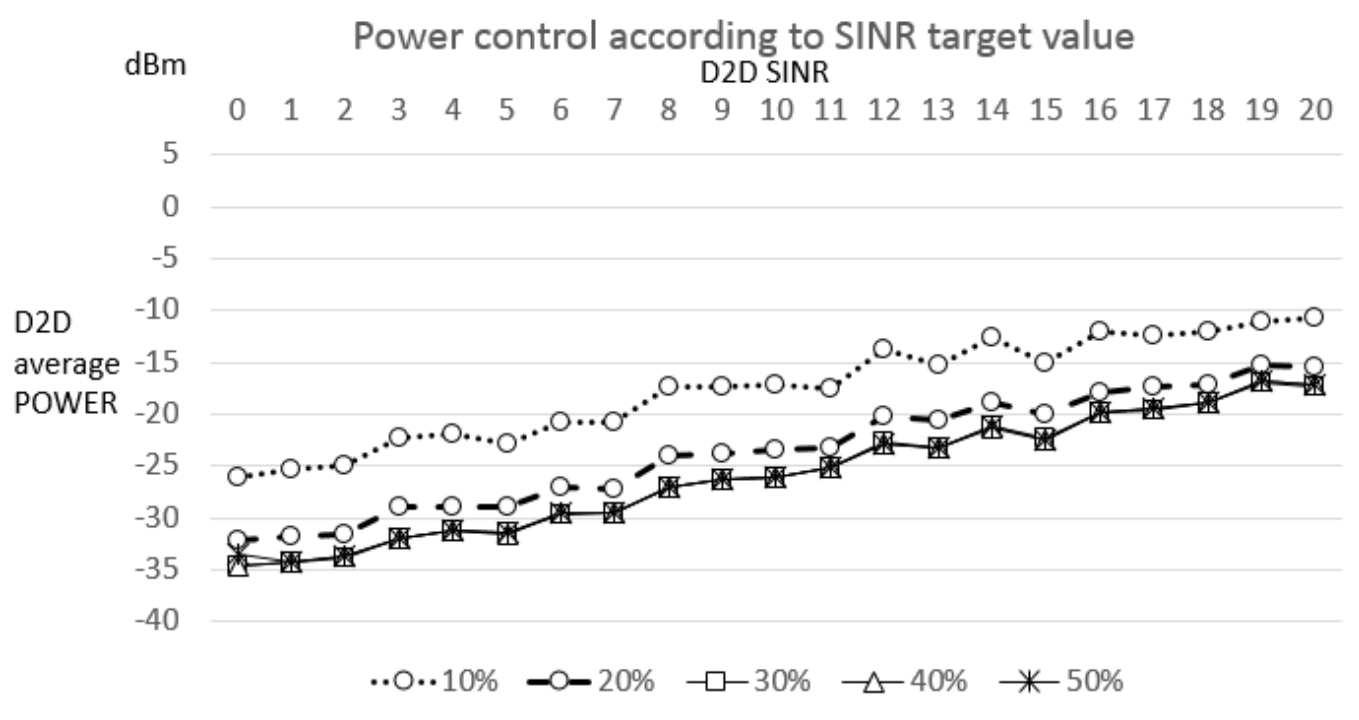

Figure 6. D2D Average with Power Control According to SINR Target Value

\section{Conclusion}

In this paper, we have identified the benefits of adding power control to the D2D resource allocation scheme. It is confirmed that the power gain is large, but the throughput is reduced overall. We will use this information to confirm that it will be available in future communications technologies. We plan to further study the use of D2D in LED communication technologies, as well as continue to analyze how resources can be managed more efficiently in D2D communication by utilizing both cellular and LED communications.

\section{Acknowledgment}

This paper is a revised and expanded version of a paper entitled [Performance Evaluation in D2D Cellular networks with power Control] presented at [S.H. Kang and J. G. Kim, Jeju, Korea and August 21, 2017].

This work was supported by Individual Basic Research Program through the Ministry of Education and National Research Foundation of Korea (NRF2017R1D1A1B03035712) and also supported by Institute for Information \& communications Technology Promotion grant funded by the Korea government (No.2017-0-00218, International Standardization Research of VLC Application Technology based LiFi and CamCom).

\section{References}

[1] K. Doppler, M. Rinne, C. Wijiting, C. B. Ribeiro and K. Hugl, "Device-to-device communication as an underlay to lte-advanced networks", IEEE Communications Magazine, vol. 47, no. 12, (2009), pp. 42 49.

[2] G. Fodor, E. Dahlman, G. Mildh, S. Parkvall, N. Reider, G. Miklos, and Z. Turanyi, "Design aspects of network assisted device-to-device communications," IEEE Communications Magazine, vol. 50, no. 3, (2012), pp. 170-177.

[3] Y. Wang, L. Tao, X. Huang, J. Shi, and N. Chi, "8-Gb/s RGBY LED-Based WDM VLC System Employing High-Order CAP Modulation and Hybrid Post Equalizer,” IEEE Photonics J., vol. 7, no. 6, (2015), pp. 1-7.

[4] L. Liang, G. Y. Li, and W. Xu, "Resource Allocation for D2D-Enabled Vehicular Communications," IEEE Transactions on Communications, vol. 65, no. 7, (2017), pp.3186-3197.

[5] M. S. Corson, R. Laroia, J. Li, V. Park, T. Richardson, and G. Tsirtsis, "Toward proximity-aware internetworking," IEEE Wireless Communications, vol. 17, no. 6, (2010). 
[6] M. Zulhasnine, C. Huang, and A. Srinivasan, "Efficient resource allocation for device-to-device communication underlaying LTE network," Wireless and Mobile Computing, Networking and Communications (WiMob), 2010 IEEE 6th International Conference on, (2010), October.

[7] H. Meshgi, D.Zhao, and R. Zheng, "Joint channel and power allocation in underlay multicast device-todevice communications," Communications (ICC), 2015 IEEE International Conference on, (2015), June.

[8] X. Li, O.S. Shin, and Y. Shin, "Resource Allocation and Power Control for Device-to-Device Communication in LTE-Advanced Based on User Area Information," The Journal of the Korean Institute of Communication Sciences, vol. 40, no. 1, (2015), pp. 32-42.

[9] A. Vavoulas, H. G. Sandalidis, T. A. Tsiftsis, and N. Vaiopoulis, "Coverage Aspects of Indoor VLC Networks," Journal of LED wave Technology, vol. 33, no. 23, (2015), pp. 4915-4921.

[10] R. Zhanh, J. Wang, Z. Wang, Z. Xu, C. Zhao, and L. Hanzo, "Visible LED communications in heterogeneous networks: Paving the way for user-centric design," IEEE Wireless Communications, vol. 22, no. 2, (2015), pp. 8-16.

[11] H. Zhang, W. Ding, J. Song, and Z. Han, "A Hierarchical Game Approach for Visible LED Communication and D2D Heterogeneous Network," Global Communications Conference (GLOBECOM), (2016), December.

[12] S. H. Kang, S. Y. Um, and J. G. Kim, "A Study on Resource Allocation Scheme for D2D Communication in Cellular Networks," Proceedings of Symposium of the Korean Institute of communications and Information Sciences, (2017).

[13] M. Rodziewicz, "Location-based mode selection and resource allocation in cellular networks with D2D underlay,” European Wireless 2015; 21th European Wireless Conference; Proceedings of, (2015), May.

\section{Authors}

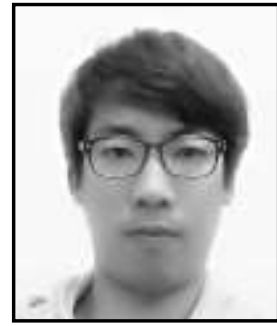

Soo Hyeong Kang, was born in Seoul, Korea on November 20, 1991. He received the B.S degrees in Electronics Engineering Department from Korea Polytechnic University in February 2016. He enrolled the M.S. degrees in Electronics Engineering Department from Korea Polytechnic University in February 2016 until present. His research interests are wireless communication, D2D (Device-toDevice) communication, MIMO.

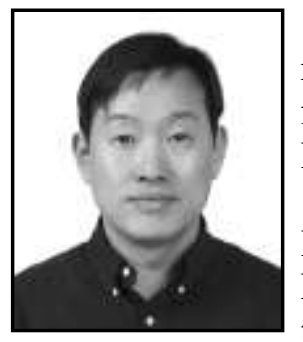

Jeong Gon Kim, was born in Seoul, Korea on May 24, 1969. He received the B.S., M.S. and Ph.D, degrees all in electrical engineering from Korea Advanced Institute of Science and Technology (KAIST), Daejeon, Korea in 1991, 1993 and 1998, respectively. From 1998 to 1999, he was the Post Doctoral Research Fellow at the University of Hawaii at Manoa, USA, from 1999-2001, he joined R\&D center of LG Telecom, Korea and is involved in IMT-2000 radio access technology development. From 2001-2003, he was also involved in 3GPP physical layer standardization, concentrating on the TDD mode in the Telecommunication Research center of Samsung Electronics. Since 2003, he is now a Professor at the Department of Electronics Engineering of Korea Polytechnic University. His research interests now include the design and performance analysis of wireless communication system, specially 5G mobile communication, MIMO, cooperative communication and WBAN based healthcare applications. 
International Journal of Grid and Distributed Computing

Vol. 11, No. 6 (2018) 